
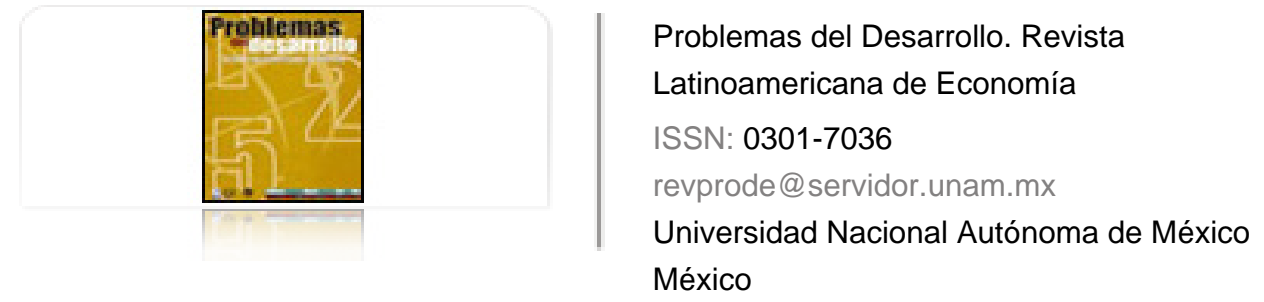

Carranco Gallardo, Zorayda; Venegas-Martínez, Francisco

Políticas fiscal y monetaria óptimas en una economía pequeña y abierta Problemas del Desarrollo. Revista Latinoamericana de Economía, vol. 40, núm. 156, enero-marzo, 2009, pp. 29-46

Universidad Nacional Autónoma de México

Distrito Federal, México

Disponible en: http://www.redalyc.org/articulo.oa?id=11820096008

- Cómo citar el artículo

- Número completo

- Más información del artículo

Página de la revista en redalyc.org

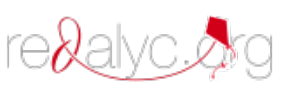

Sistema de Información Científica

Red de Revistas Científicas de América Latina, el Caribe, España y Portugal Proyecto académico sin fines de lucro, desarrollado bajo la iniciativa de acceso abierto 


\title{
POLÍTICAS FISCAL Y MONETARIA ÓPTIMAS EN UNA ECONOMÍA PEQUEÑA Y ABIERTA
}

\author{
Zorayda Carranco Gallardo* \\ Francisco Venegas-Martínez**
}

Fecha de recepción: 4 de agosto de 2008. Fecha de aceptación: 18 de noviembre de 2008.

\section{Resumen}

Esta investigación desarrolla un modelo de una economía pequeña y abierta, con perfecta movilidad de capital, que permite examinar las acciones del gobierno en materia fiscal y monetaria que son compatibles con los planes óptimos del sector privado. El modelo propuesto considera a un individuo representativo con previsión perfecta. El resultado central es que las políticas fiscal y monetaria consistentes con los planes óptimos de consumidores y firmas racionales no deben generar distorsiones.

Palabras clave: política fiscal, política monetaria, análisis conjunto de políticas fiscal $y$ monetaria.

* Profesora-investigadora en el Departamento de Economía de la UAM-Azcapotzalco. Correo electrónico: zorayda.carranco@gmail.com.mx.

** Profesor-investigador en la Escuela Superior de Economía del IPN. Correo electrónico: fvenegas1111@yahoo.co.mx. 


\section{0}

\section{Abstract}

This study develops a model of a small, open economy, with perfect capital mobility, which makes it possible to examine the actions of the government in the fiscal and monetary areas, compatible with optimal plans in the private sector. The proposed model considers a representative individual with perfect foresight. The key result is that fiscal and monetary policies consistent with the optimal plans of consumers and rational firms should not generate distortions.

Key words: fiscal policy, monetary policy, joint analysis of fiscal and monetary policies.

\section{Résumé}

Cette recherche développe un modèle d'économe petite et ouverte, avec une parfaite mobilité de capital, qui permet d'examiner les actions du gouvernement en matière fiscale et monétaire qui sont compatibles avec les plans optimums du secteur privé. Le modèle proposé considère un individu représentatif avec prévision parfaite. Le résultat central est que les politiques fiscale et monétaire congruentes avec les plans optimums des consommateurs et firmes nationales ne doivent pas engendrer de distorsions.

Mots clés: politique fiscale, politique monétaire, analyse conjointe de politiques fiscale et monétaire.

\section{Resumo}

Esta pesquisa desenvolve um modelo de uma economia pequena e aberta, com perfeita movimentação de capital, que permite examinar as ações do governo na matéria fiscal e monetária que são compatíveis com os planos ótimos do setor privado. $O$ modelo proposto considera a um individuo representativo com previsão perfeita. $O$ resultado central é que a política fiscal e monetária consistentes com os planos ótimos dos consumidores e empresas racionais não deve gerar distorções.

Palavras chave: política fiscal, política monetária, análise conjunta de políticas fiscal e monetária.

\section{Desarrollo}




\section{Introducción}

diseño y la aplicación de las políticas fiscal y monetaria han sido un tema re-
levante y de continua discusión, ya que el papel que desempeña el gobierno en
las actividades económicas no resulta claro, principalmente por las distorsiones que puede generar en las decisiones de los agentes económicos (productores y consumidores). La literatura sobre este tema es extensa.

Varios autores han abordado esta discusión al evaluar los efectos de las políticas económicas sobre las funciones de producción y utilidad. Uno de los trabajos destacados sobre este tema es el de Barro (1990), en donde el gasto afecta la producción de las firmas y la satisfacción de los individuos. Por su parte, Phelps (1973) se concentra en el tradeoff entre la recaudación de un impuesto sobre la renta y el impuesto inflacionario. En este estudio Phelps muestra que un impuesto inflacionario tiene efectos directos sobre la liquidez. Por último, Lockwood (1997) analiza la eficacia de la política monetaria para contener el desempleo persistente y concluye que entre mayor sea el desempleo, más incentivos tiene el gobierno para intervenir con medidas monetarias, pero ello obviamente genera distorsiones en el nivel de la inflación.

Por otro lado, Turnovsky (1999) analiza los efectos de la política fiscal sobre el crecimiento y la elasticidad de la oferta de trabajo. Asimismo, Turnovsky (2000) determina una política fiscal óptima y evalúa su efecto sobre el bienestar. En otro trabajo (1998) estudia una economía con riesgo y obtiene las políticas fiscal y monetaria óptimas, las cuales son independientes del tipo de bono o del tipo de impuesto que el gobierno utilice para financiarse. Por último, Turnovsky (2002) analiza los efectos de la política fiscal sobre la acumulación de capital en el largo plazo.

Este trabajo se concentra en el desarrollo de un modelo que coordina de manera óptima las acciones de las autoridades fiscal y monetaria con las de los consumidores y productores. Es decir, el modelo permite coordinar el manejo de los instrumentos de política fiscal y monetaria con las decisiones de consumo, portafolio y producción.

Una característica distintiva del modelo propuesto es que utiliza funciones explícitas de utilidad y producción de los agentes, lo cual permite obtener soluciones analíticas en el equilibrio, facilitando con ello tanto la obtención de los resultados como la interpretación de los mismos en cuanto a los aspectos esenciales de coordinación entre los sectores público y privado. Bajo estas condiciones, el modelo es una aplicación directa de la propuesta de Turnovsky (1987). Uno de los principales resultados es que las políticas fiscal y monetaria que son consistentes con los planes óptimos de consumidores y firmas racionales no deben generar distorsiones de liquidez en los agentes. 
Este trabajo se organiza de la siguiente manera: primero se lleva a cabo el planteamiento de la estructura de la economía en estudio. Luego se establecen las condiciones de equilibrio macroeconómico. Después se introduce el problema de decisión del gobierno, se determinan de manera óptima sus variables de decisión y se analizan las políticas monetaria y fiscal correspondientes. Al final se presentan las conclusiones.

\section{Estructura de la economía}

Considere una economía poblada por consumidores que también son productores con preferencias, dotaciones y tecnologías idénticas. Estos individuos viven para siempre y desean aumentar su satisfacción por un bien genérico de consumo (el supuesto de vida infinita se puede reemplazar por un padre que se interesa por elevar la satisfacción de sus hijos, nietos, bisnietos y demás descendientes). La economía en cuestión es la de un país que no puede influir en los precios externos y se mantendrá por siempre en esta condición, es decir, su comportamiento será permanentemente el de una economía tomadora de precios.

En conclusión, se trata de una economía pequeña y abierta. Se supone que la economía produce y consume un solo bien comerciable internacionalmente libre de barreras y restricciones arancelarias. El nivel general de precios domésticos, $P_{t}$, está determinado por la condición de paridad de poder de compra, la cual está dada por $P_{t}=P_{t}^{*} E_{t}$ donde $P_{t}^{*}$ es el precio en moneda extranjera del bien en el resto del mundo y $E_{t}$ es el tipo de cambio nominal. La condición de paridad del poder de compra se puede reescribir como

$$
\frac{\mathrm{d}}{\mathrm{d} t} \ln \left(P_{t}\right)=\frac{\mathrm{d}}{\mathrm{d} t} \ln \left(P_{t}^{*}\right)+\frac{\mathrm{d}}{\mathrm{d} t} \ln \left(E_{t}\right)
$$

0

$$
\frac{\mathrm{d} P_{t}}{P_{t}} \frac{1}{\mathrm{~d} t}=\frac{\mathrm{d} P_{t}^{*}}{P_{t}} \frac{1}{\mathrm{~d} t}+\frac{\mathrm{d} E_{t}}{E_{t}} \frac{1}{\mathrm{~d} t}
$$

equivalentemente,

$$
\pi_{t}=\pi_{t}^{*}+\varepsilon_{t},
$$

donde $\pi_{t}$ es la tasa de inflación en la economía doméstica, $\pi_{t}^{*}$ es la tasa de inflación del resto del mundo, la cual, sin pérdida de generalidad, se supone constante, 
$\pi_{t}^{*}=\pi^{*}$ y $\varepsilon_{t}$ es la tasa de depreciación del tipo de cambio. Si se supone que los individuos tienen previsión perfecta, entonces $\pi_{t} \equiv \pi_{t}^{e}$, es decir, la tasa de inflación observada es igual a la tasa de inflación esperada.

En esta economía los residentes pueden tener dos tipos de activos: moneda doméstica y bonos internacionalmente negociables denominados en moneda extranjera (por ejemplo, dólar). Bajo condiciones de arbitraje internacional se supone también que se satisface la paridad de tasas de interés, es decir:

$$
r_{t}=r_{t}^{*}+\varepsilon_{t}
$$

donde $r_{t}$ es la tasa de interés real doméstica y $r_{t}^{*}$ es la tasa de interés real internacional, la cual, sin menoscabo de generalidad, se supone constante, $r_{t}^{*}=r^{*}$. Suponga que la tasa subjetiva de descuento (o tasa subjetiva intertemporal) $\rho$ del consumidor representativo coincide con la tasa de interés real internacional. Por supuesto, esta coincidencia se debe únicamente a la casualidad, no existe otra razón para suponer que el valor de estos parámetros sea el mismo.

El propósito de incluir dicho supuesto consiste en definir de manera correcta el estado estacionario en las variables relevantes de la economía; véase, por ejemplo, Venegas-Martínez (2000). Observe que si se combinan las condiciones de paridad de poder de compra y de tasas de interés, la tasa subjetiva de descuento satisface:

$$
\rho=r_{t}-\pi_{t}^{e}=r^{*}-\pi^{*}
$$

Observe que las tasas de interés real doméstica y extranjera son idénticas, con lo cual se tiene perfecta movilidad de capitales. Note, por último, que la tasa de interés real doméstica satisface $r_{t}=r^{*}-\pi^{*}+\pi_{t}^{e}$.

Ahora se supone que el consumidor además de obtener satisfacción por el bien de consumo, deriva satisfacción por la tenencia de saldos monetarios reales (por sus servicios de liquidez), por el ocio (tiempo de esparcimiento que destina al trabajo) y por el gasto de gobierno (por ejemplo, por la construcción de carreteras para transportar bienes finales a puntos de demanda, lo cual hace felices a los consumidores). Por ello se supone que el agente representativo tiene una función de utilidad de la forma:

$$
u\left(c_{t}, l_{t}, m_{t}, g_{t}\right)=\ln c_{t}-\beta \ln l_{t}+\gamma \ln m_{t}+\delta \ln g_{t}
$$

donde $c_{t}$ es el consumo, $l_{t}$ representa el trabajo, $m_{t}$ son los saldos reales y $g_{t}$ es el gasto de gobierno. Todas las constantes, $\beta, \gamma$ y $\delta$ son positivas y miden el impacto de cada 
variable económica sobre la utilidad del individuo. Observe que la función de utilidad propuesta es cóncava en $c_{t}, m_{t}$ y $g_{t}$ y convexa en $l_{t}$. Se supone, y es evidente hacerlo así, que aunque el agente representativo obtiene satisfacción por el gasto público, no tiene el control sobre éste.

En una segunda etapa (de un juego de dos fases) el gobierno decidirá sobre el nivel del gasto, así como de otros instrumentos de política fiscal y monetaria. Así pues, el consumidor tendrá que elegir $c_{t}, b_{t}, l_{t}$ y $m_{t}$ para aumentar su utilidad sujeta a la siguiente restricción presupuestal intertemporal:

$$
\dot{m}_{t}+\dot{b}_{t}=\left(1-\tau_{t}\right)\left(w_{t} l_{t}+\Pi_{t}\right)+\left(r^{*}-\pi^{*}\right) b_{t}-\left(\pi^{*}+\varepsilon_{t}\right) m_{t}-c_{t}-T_{t}
$$

donde $w_{t}$ es el salario real (poder de compra), $\Pi_{t}$ son los beneficios reales por la actividad como productor, $\tau$ es la tasa constante de impuestos a los ingresos (impuesto sobre la renta) y $T_{t}$ es un impuesto en suma fija. Por tanto, la riqueza del agente representativo está en función de sus percepciones salariales y sus beneficios como empresario después de un impuesto sobre la renta, más el interés generado por la tenencia de bonos, menos la depreciación de sus saldos reales y menos un impuesto en suma fija (en el caso de ser positivo sería una transferencia del gobierno). Evidentemente, el aumento en las variables de política fiscal genera una disminución en el ingreso, mientras que el aumento en rendimiento de los bonos ocasiona un incremento en el ingreso.

Dichos ingresos son destinados, por parte del individuo, al consumo corriente, a la tenencia de bonos y a mantener saldos reales. Dado lo anterior el consumidor racional competitivo (tomador de precios) se enfrenta al siguiente problema de crecimiento intertemporal:

$$
\text { Maximizar } \quad U=\int_{0}^{\infty}\left(\ln c_{t}-\beta \ln l_{t}+\gamma \ln m_{t}+\delta \ln g_{t}\right) e^{-\rho t} \mathrm{~d} t
$$

sujeto a

$$
\dot{m}_{t}+\dot{b}_{t}+c_{t}=\left(1-\tau_{t}\right)\left(w_{t} l_{t}+\Pi_{t}\right)+\left(r^{*}-\pi^{*}\right) b_{t}-\left(\pi^{*}+\varepsilon_{t}\right) m_{t}-T_{t}
$$

Se consideran las siguientes condiciones iniciales sobre la tenencia de saldos reales y sobre la tenencia de bonos: 


$$
m_{0}=\frac{M_{0}}{P_{0}}=\frac{M_{0}}{P_{0}^{*} E_{0}} \quad \text { y } \quad b_{0}=\frac{E_{0} B_{0}}{P_{0}^{*} E_{0}}=\frac{B_{0}}{P_{0}^{*}}
$$

donde $M_{0}$ es el acervo inicial de dinero nominal; $B_{0}$ es el stock nominal de bonos y $P_{0}$ y $P_{0}{ }^{*}$ son los niveles iniciales de precios doméstico y externo, respectivamente, y $E_{0}$ es el tipo de cambio nominal inicial expresado en unidades de moneda doméstica por unidad de moneda externa. El agente toma como dadas las variables $g_{t}, T_{t}, \varepsilon_{t}, p^{*}$, $\Pi_{t}, w_{t}, r^{*}, E_{0}, P_{0}^{*}$ y $P_{0}$, para determinar sus planes óptimos sobre $c_{t}, l_{t}, m_{t} \mathrm{y} b_{t}$.

\section{Condiciones de equilibrio macroeconómico}

En esta sección se resuelven los problemas de decisión de consumidores y productores. Es decir, se obtienen las trayectorias óptimas del consumo, del trabajo y de la tenencia de saldos reales de los individuos, ${ }^{1}$ así como los planes óptimos de producción.

Dadas las condiciones (7) y (8), en donde se ha supuesto que $\rho=r_{t}-\pi_{t}^{e}=r^{*}-\pi^{*}$, se supone también que al mismo tiempo los individuos son consumidores y empresarios, de manera tal que reciben los beneficios generados por la actividad productiva. Dichos beneficios consisten en sus ventas totales menos sus costos, es decir

$$
\Pi_{t}=f\left(l_{t}\right)-w_{t} l_{t}
$$

donde $f\left(l_{t}\right)$ es una función de producción neoclásica, es decir, $f(0)=0$ (no existe la generación instantánea de bienes), $f^{\prime}\left(l_{t}\right)>0$ (cualquier cantidad de trabajo $l_{t}>0$ tiene la capacidad de producir bienes) y $f^{\prime \prime}\left(l_{t}\right)<0$ (ley de rendimientos marginales decrecientes). En particular se supondrá que $f\left(l_{t}\right)=A l_{t}^{\phi}, A>0,0<\phi<1$. La condición de primer orden (condición necesaria) para una solución interior del problema de aumentar el flujo esperado de beneficios,

$$
\text { Maximizar } \int_{0}^{\infty} \Pi_{t} e^{-(r-\pi) t} \mathrm{~d} t
$$

es que el producto marginal del trabajo es igual al salario real, es decir,

$$
A \phi l_{t}^{\phi-1}=w_{t}
$$

1 Algunas variantes estocásticas de este modelo pueden ser encontradas en Venegas-Martínez (2001, 2206 y 2008). 
Al sustituir la ecuación (10) en (8), se tiene que

$$
\left(1-\tau_{t}\right) A l_{t}^{\phi}+\left(r^{*}-\pi^{*}\right) b_{t}-\left(\pi^{*}+\varepsilon_{t}\right) m_{t}-T_{t}-\dot{m}_{t}-\dot{b}_{t}-c_{t}=0
$$

Lo anterior conduce a que el impuesto al ingreso gravará directamente a la producción, al incluir de manera directa la función de producción dentro de la restricción presupuestal del consumidor. Por tanto, el agente representativo se enfrenta al siguiente problema de optimización:

$$
\begin{aligned}
L= & \left(\ln c_{t}-\beta \ln l_{t}+\gamma \ln m_{t}+\delta \ln g_{t}\right) e^{-\left(r^{*}-\pi^{*}\right) t} \\
& +\mu_{t} e^{-\left(r^{*}-q\right) t}\left[\left(1-\tau_{t}\right) A l_{t}^{\phi}+\left(r^{*}-\pi^{*}\right) b_{t}-\left(\pi^{*}+\varepsilon_{t}\right) m_{t}-T_{t}-\dot{m}_{t}-\dot{b}_{t}-c_{t}\right]
\end{aligned}
$$

en donde $\mu_{t}$ es el multiplicador de Lagrange asociado a la restricción presupuestal. Las condiciones de primer orden, en este caso, son

donde

$$
\begin{aligned}
& \frac{\partial L}{\partial c_{t}}=\frac{1}{c_{t}}-\mu_{t}=0 \\
& \frac{\partial L}{\partial l_{t}}=-\frac{\beta}{l_{t}}+\mu_{t}\left(1-\tau_{t}\right) A \phi l_{t}^{\phi-1}=0 \\
& \frac{\partial L}{\partial m_{t}}=\frac{\gamma}{m_{t}}-\mu_{t}\left(\pi^{*}+\varepsilon_{t}\right)=0
\end{aligned}
$$

$$
\dot{\mu}_{t}=\frac{\partial \dot{m}_{t}}{\partial m_{t}}
$$

Es decir, el multiplicador de Lagrange es igual a la utilidad marginal del consumo. Si se supone que $\dot{\mu}_{t}=0$, entonces $\mu_{t} \equiv \mu$ es una constante y por tanto el consumo también lo es. Este resultado es consecuencia directa de suponer coincidencia entre la tasa subjetiva de descuento y la tasa de interés nominal internacional. Para garantizar las condiciones iniciales se tiene la siguiente condición de transversalidades:

$$
\lim _{t \rightarrow \infty} \mu m_{t} e^{-\left(r^{*}-\pi^{*}\right) t}=\lim _{t \rightarrow \infty} \mu b_{t} e^{-\left(r^{*}-\pi^{*}\right) t}=0
$$

es decir, esta condición nos garantiza que tanto los saldos reales como es el stock de bonos domésticos en el límite se agotan. 
Por otra parte, el gobierno especifica la política monetaria con una tasa constante $\theta$, de manera que la masa monetaria evoluciona de acuerdo con

$$
\dot{m}_{t}=\left(\theta-\pi^{*}-\varepsilon_{t}\right) m_{t}
$$

La trayectoria de los saldos reales está en función de dicha tasa de crecimiento, la cual se ve disminuida por la inflación. La restricción presupuestal del gobierno está dada por

$$
g_{t}=\dot{a}_{t}-\left(r^{*}-\pi^{*}\right) a_{t}+\tau\left(w_{t} l_{t}+\Pi_{t}\right)+\theta m_{t}+T_{t}
$$

donde $a_{t}$ son los bonos que emite el gobierno. En consecuencia, la trayectoria de los bonos internacionalmente comerciables, $a_{t}$, que emite el gobierno es

$$
\dot{a}_{t}=g_{t}+\left(r^{*}-\pi^{*}\right) a_{t}-\tau\left(w_{t} l_{t}+\Pi_{t}\right)-\theta m_{t}-T_{t}
$$

El crédito neto de la economía está definido mediante la igualdad $n_{t}=b_{t}-a_{t}$, ello constituye, por tanto, una restricción de la economía. Consecuentemente, la trayectoria del crédito neto está dado por

$$
\dot{n}_{t}=A l_{t}^{\phi}-c_{t}-g_{t}+\left(r^{*}-\pi^{*}\right) n_{t}
$$

Por tanto, el sistema de ecuaciones que determina el equilibrio está dado por

$$
\begin{aligned}
& \frac{1}{c_{t}}=\mu \\
& \frac{\beta}{l_{t}}=\mu(1-\tau) A \phi l_{t}^{\phi-1} \\
& \frac{\gamma}{m_{t}}=\mu\left(r^{*}+\varepsilon_{t}\right) \\
& \dot{m}_{t}=\left(\theta-\pi^{*}-\varepsilon_{t}\right) m_{t} \\
& \dot{b}_{t}=\left(r^{*}-\pi^{*}\right) b_{t}+(1-\tau) A l_{t}^{\phi}-c_{t}-T_{t}-\theta m_{t} \\
& \dot{n}_{t}=\left(r^{*}-\pi^{*}\right) n_{t}+A l_{t}^{\phi}-c_{t}-g_{t}
\end{aligned}
$$

El equilibrio está formado por el sistema de ecuaciones que consideran las restricciones y condiciones de primer orden del consumidor-productor representativo. 
A partir de este sistema se obtienen los niveles de equilibrio de $c_{t}, l_{t}$ y $\varepsilon_{t}$, los cuales están dados por:

$$
\begin{aligned}
& c_{t}=\frac{1}{\mu} \\
& l_{t}=\frac{\beta}{A \phi l_{t}^{\phi-1}(1-\tau) \mu} \\
& \varepsilon_{t}=\frac{\gamma}{m_{t} \mu}-r^{*}
\end{aligned}
$$

A partir de las ecuaciones (23) se sabe que la trayectoria del consumo depende de manera inversa de la tasa de crecimiento de los saldos reales, es decir, a medida de que aumenta la tenencia de saldos reales, disminuye el consumo. Por su parte, el ocio depende inversamente del valor monetario del ingreso disponible y de forma directa de la elasticidad intertemporal de sustitución del ocio, por lo que un incremento en el ingreso o una disminución en el impuesto al ingreso ocasiona una caída en el ocio. Por último, el tipo de cambio depende de la elasticidad intertemporal de sustitución de los saldos reales y de la tasa de interés real. Bajo estas condiciones las trayectorias óptimas del consumo y del trabajo son en cada caso:

Gráfica 1

Trayectoria óptima del consumo

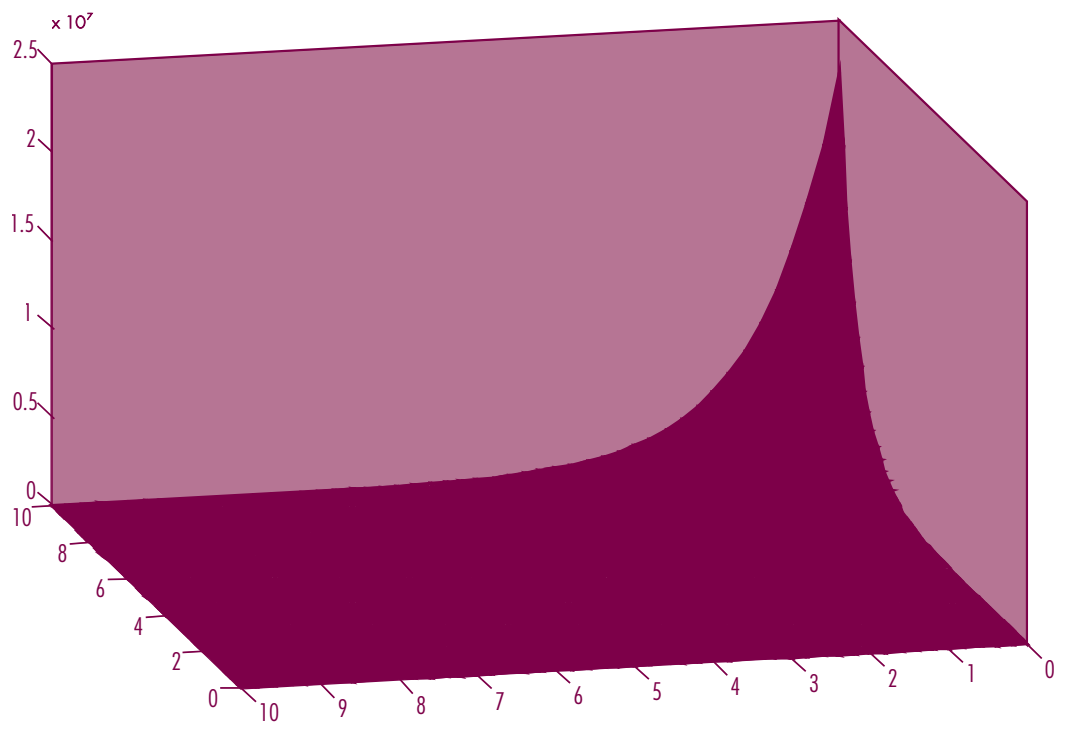

Fuente: Elaboración propia. 
Grafica 2

Trayectoria óptima del trabajo

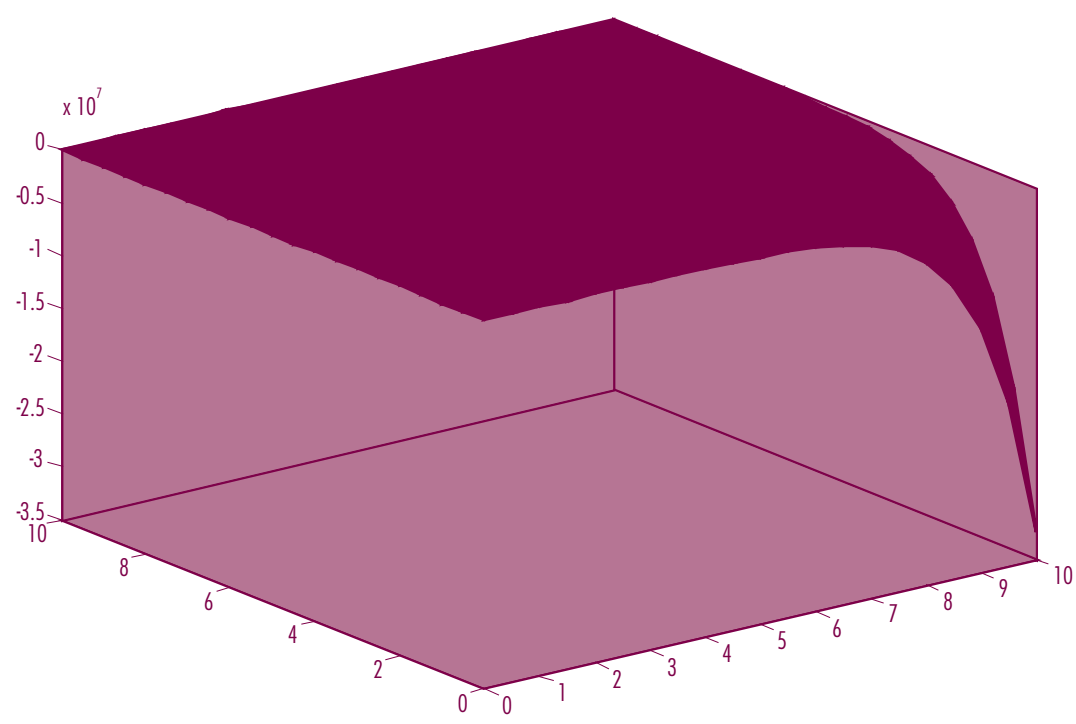

Fuente: Elaboración propia.

Respecto de la depreciación (apreciación) del tipo de cambio, está en función de los saldos reales y de la tasa de interés real internacional. En virtud de (22), si sustituye la expresión de $\varepsilon_{t}$ en la ecuación para $\dot{m}_{t}$, se tiene:

$$
\dot{m}_{t}=\left[\theta-\pi^{*}-\left(\frac{\gamma}{m_{t} \mu}-r^{*}\right)\right] m_{t}
$$

En lo que sigue se supone que los saldos reales permanecen en un estado estacionario, es decir $\dot{m}_{t} \equiv 0$. Si se utiliza este supuesto con (22), se cumple que $\theta=\pi^{*}+\varepsilon_{t^{*}}$. Observe que $m_{t}$ es constante y, en consecuencia, $c_{t}, l_{t}$ y $\varepsilon_{t}$ también lo son. En lo que sigue estas constantes se denotarán mediante $\bar{m}, \bar{c}, \bar{\varepsilon}$ y $\bar{l}$. De este modo, si se supone, por el momento, un nivel constante del impuesto de suma fija $T_{t} \equiv \bar{T}$, se puede encontrar que la trayectoria de los bonos en manos del consumidor está dada por:

$$
\dot{b}_{t}=\left(r^{*}-\pi^{*}\right) b_{t}+(1-\tau) A \bar{l}^{\phi}-\bar{c}-\theta \bar{m}-\bar{T}
$$

La solución de esta ecuación diferencial no homogénea de orden 1 está dada por: 


$$
b_{t}=e^{\left(r^{*}-\pi^{*}\right) t}\left[b_{0}+\left(\frac{(1-\tau) A \bar{l}^{\phi}-\bar{c}-\theta \bar{m}-\bar{T}}{r^{*}-\pi^{*}}\right)\left(1-e^{-\left(r^{*}-\pi^{*}\right) t}\right)\right]
$$

Por tanto, dada la ecuación (9), las condiciones óptimas iniciales de los bonos satisfacen la siguiente relación:

$$
\left(r^{*}-\pi^{*}\right) b_{0}+(1-\tau) A \bar{l}^{\phi}-\bar{c}-\theta \bar{m}-\bar{T}=0
$$

Análogamente sucede con la trayectoria del crédito neto de la economía si se supone, por el momento, un nivel constante del gasto público, $g_{t} \equiv \bar{g}$ :

$$
\dot{n}_{t}=\left(r^{*}-\pi^{*}\right) n_{t}+A \bar{l}^{\phi}-\bar{c}-\bar{g}
$$

La solución de la ecuación diferencial anterior es

$$
n_{t}=e^{\left(r^{*}-\pi^{*}\right) t}\left[n_{0}+\left(\frac{A \bar{l}^{\phi}-\bar{c}-\bar{g}}{r^{*}-\pi^{*}}\right)\left(1-e^{-\left(r^{*}-\pi^{*}\right) t}\right)\right]
$$

La ecuación anterior nos muestra la trayectoria del crédito neto de la economía, el cual depende de manera positiva de sus condiciones iniciales y del ingreso, así como del consumo, del gasto de gobierno, de la inflación internacional y de la tasa de interés real internacional, de forma negativa. En consecuencia, el crédito neto inicial de la economía doméstica, por la condición de transversalidad del crédito doméstico, es:

$$
\left(r^{*}-\pi^{*}\right) n_{0}+\left[A \bar{l}^{\phi}-\bar{c}+\bar{g}\right]=0
$$

La ecuación anterior puede expresarse en términos de bonos domésticos y negociables, por lo que se tiene la diferencia de los bonos que retienen los connacionales y los vendidos al exterior:

$$
\left(r^{*}-\pi^{*}\right)\left(b_{0}-a_{0}\right)+\left[A \bar{l}^{\phi}-\bar{c}+\bar{g}\right]=0
$$

Bajo estas nuevas condiciones, el equilibrio en el sector privado se ha modificado de la siguiente manera:

\section{Desarrollo}




$$
\begin{aligned}
& \frac{1}{\bar{c}}=\mu \\
& \frac{\beta}{\bar{l}}=\mu(1-\tau) A \phi \bar{l}^{\phi-1} \\
& \frac{\gamma}{\bar{m}}=\mu\left(r^{*}+\bar{\varepsilon}\right) \\
& \left(r^{*}-\pi^{*}\right) b_{0}+(1-\tau) A \bar{l}^{\phi}-\bar{c}-\bar{T}-\theta \bar{m}=0 \\
& \left(r^{*}-\pi^{*}\right)\left(b_{0}-a_{0}\right)+A \bar{l}^{\phi}-\bar{c}-\bar{g}=0
\end{aligned}
$$

En este sistema de ecuaciones el agente representativo ha decidido sus niveles de consumo, la cantidad óptima de trabajo y lo que desea mantener en saldos reales frente a la opción de poseer bonos. Además, se agregan los niveles óptimos iniciales de los bonos domésticos y del crédito neto, que dependen de la tasa de interés real, de la inflación internacional, del ingreso y del consumo. Además los bonos están supeditados al impuesto de suma fija y por su parte el crédito al nivel del gasto de gobierno.

\section{Comportamiento del gobierno}

A partir de las condiciones de equilibrio del sector privado dadas por el apartado anterior, el gobierno ahora formulará sus decisiones óptimas sobre las trayectorias de las política y monetaria. En este problema se incorporan las decisiones del individuo representativo y las restricciones de la economía, suponiendo la coincidencia de la tasa subjetiva de descuento del individuo y la tasa de interés nominal internacional, así como el supuesto de estado estacionario de la política monetaria. Por tanto, el gobierno tiene que decidir cuáles son los niveles óptimos de impuesto al ingreso, tasa de expansión monetaria y de gasto fiscal, los cuales se coordinarán con las decisiones privadas. Para ello, el lagrangiano asociado al problema de optimización del gobierno, tomando en cuenta el equilibrio en el sector privado, es:

$$
\begin{aligned}
\bar{L}= & \left(\ln c_{t}-\beta \ln l_{t}+\gamma \ln m_{t}+\delta \ln g_{t}\right) e^{-\left(r^{*}-\pi^{*}\right) t} \\
& -v_{1} e^{-\left(r^{*}-\pi^{*}\right) t}\left(\frac{1}{c_{t}}-\mu\right)-v_{2} e^{-\left(r^{*}-\pi^{*}\right) t}\left[\mu(1-\tau) A \phi l_{t}^{\phi-1}-\frac{\beta}{l_{t}}\right] \\
& -v_{3} e^{-\left(r^{*}-\pi^{*}\right) t}\left[\frac{\gamma}{m_{t}}-\mu\left(r^{*}+\theta-\pi^{*}\right)\right] \\
& +v_{4} e^{-\left(r^{*}-\pi^{*}\right) t}\left[\left(r^{*}-\pi^{*}\right) b_{0}+(1-\tau) A l_{t}^{\phi}-c_{t}-T_{t}-\theta m_{t}\right] \\
& +v_{5} e^{-\left(r^{*}-\pi^{*}\right) t}\left[\left(r^{*}-\pi^{*}\right)\left(b_{0}-a_{0}\right)+A l_{t}^{\phi}-c_{t}-g_{t}\right]
\end{aligned}
$$


Dado que el problema está sujeto al equilibrio obtenido en la sección anterior, es importante señalar que $v_{i}$, con $i=1, \ldots, 5$, son los multiplicadores de Lagrange asociados a las restricciones correspondientes. Las condiciones de primer orden se pueden dividir en dos grupos: el primero son las variables de sector privado, $c_{t}, l_{t} \mathrm{y}$ $m_{t}$; y el segundo lo componen las variables de política fiscal y monetaria, $\theta, T_{t}, \tau$ y $g_{t}$. En consecuencia, el primer grupo queda como

$$
\begin{aligned}
& \frac{\partial \bar{L}}{\partial c_{t}}=\frac{1}{c_{t}}-\frac{v_{1}}{c_{t}^{2}}-v_{4}-v_{5}=0 \\
& \frac{\partial \bar{L}}{\partial l_{t}}=-\frac{\beta}{l_{t}}-v_{2}\left[\mu(1-\tau) A \phi(\phi-1) l_{t}^{\phi-2}+\frac{\beta}{l_{t}^{2}}\right]+v_{4}\left[(1-\tau) A \phi l_{t}^{\phi-1}\right]+v_{5} A \phi l_{t}^{\phi-1}=0 \\
& \frac{\partial \bar{L}}{\partial m_{t}}=\frac{\gamma}{m_{t}}+v_{3}\left[\frac{\gamma}{m_{t}^{2}}\right]-v_{4} \theta=0 \\
& \frac{\partial \bar{L}}{\partial \mu}=v_{1}-v_{2}(1-\tau) A \phi l_{t}^{\phi-1}+v_{3}\left(r^{*}+\theta-\pi^{*}\right)=0
\end{aligned}
$$

Las condiciones de primer orden para el grupo de variables de política son

$$
\begin{aligned}
& \frac{\partial \bar{L}}{\partial \theta}=v_{3} \mu-v_{4} m_{t}=0 \\
& \frac{\partial \bar{L}}{\partial T_{t}}=-v_{4}=0 \\
& \frac{\partial \bar{L}}{\partial \tau}=v_{2} A \phi l_{t}^{\phi-1} \mu-v_{4} A l_{t}^{\phi}=0 \\
& \frac{\partial \bar{L}}{\partial g_{t}}=\frac{\delta}{g_{t}}-v_{5}=0
\end{aligned}
$$

Del grupo de ecuaciones dado en (35) se deduce que los saldos reales seguirán creciendo a una tasa constante, un incremento en el impuesto de suma fija tiene un efecto distorsionador sobre el equilibrio, el impuesto al ingreso está condicionado a la productividad marginal de los factores que dependen de la producción y el gasto de gobierno está supeditado de manera inversa a su elasticidad intertemporal de sustitución. La dinámica de las decisiones de gobierno será tomada en función del primer grupo de condiciones y el segundo grupo servirá para identificar qué tipo de instrumento de política se está utilizando. Por tanto, cada variable de política fiscal generará un tipo de equilibrio distinto y tendrá repercusiones en la coordinación de la política monetaria.

\section{Desarrollo}




\section{Equilibrio por medio del impuesto al ingreso}

Otro instrumento comúnmente utilizado de política fiscal es el impuesto al ingreso. Como su nombre lo indica éste graba directamente el ingreso, ya sea el generado mediante el trabajo, es decir el salario, o el que se percibe por medio de la actividad productiva, es decir los beneficios. En esta sección se busca el equilibrio mediante $\tau$, el impuesto al ingreso, para ello suponemos que es constante, con lo que se demuestra que en una economía pequeña y abierta este equilibrio lleva a un tradeoff entre el impuesto a la inflación y el impuesto al ingreso. Por tanto, el equilibrio se conforma utilizando la condición de primer orden referente a $\tau$ y su acción en $\theta$. En este caso $v_{3}$ $\mathrm{y} v_{4}$ son diferentes de cero $\mathrm{y}$, por tanto, el equilibrio es

$$
\begin{gathered}
{\left[\frac{1}{c_{t}} A \phi l_{t}^{\phi-1}+\frac{\beta}{l_{t}}\right]-v_{1} \frac{1}{c_{t}^{2}} A \phi l_{t}^{\phi-1}-v_{2}\left[\frac{\beta}{l_{t}^{2}}+\frac{A \phi(\phi-1) l_{t}^{\phi-2}(1-\tau)}{c_{t}}\right]-v_{4} \tau A \phi l_{t}^{\phi-1}=0} \\
\frac{\gamma}{m_{t}}-v_{3} \frac{\gamma}{m_{t}^{2}}-\theta v_{4}=0 \\
v_{1}-v_{2} A \phi l_{t}^{\phi-1}(1-\tau)+v_{3}\left(r^{*}+\theta-\pi^{*}\right)=0 \\
v_{3} \mu-m v_{4}=0 \\
v_{2} A \phi l_{t}^{\phi-1} \mu-v_{4} A l_{t}^{\phi}=0
\end{gathered}
$$

El conjunto de ecuaciones establecidas (36-40) conforman el equilibrio general de la economía, es decir, la compatibilidad de las decisiones de los sectores privado y el público, ya que el gobierno incorpora a sus medidas de políticas fiscal y monetaria las decisiones óptimas del consumidor, con la finalidad de no crear distorsiones en el consumo, el ocio y en la tenencia de saldos reales de los individuos. A partir de este sistema de ecuaciones y conjuntamente con (32) se obtienen los valores para $v_{3}$ y $v_{4}$ :

$$
\begin{aligned}
& v_{3}=\frac{\tau\left[A \phi l_{t}^{\phi-1}\right]^{2} \gamma}{m_{t}^{2}\left\{\left[\frac{A^{2} \phi(\phi-1) l_{t}^{2 \phi-2}(1-\tau)+\tau\left[A \phi l_{t}^{\phi-1}\right]^{2}}{c_{t}}\right]-\left[\frac{A l_{t}^{\phi}(1-\tau)-m_{t} \theta}{c_{t}^{2}}\right]-\left[\frac{A l_{t}^{\phi} \beta}{l_{t}^{2}}\right]\right\}} \\
& v_{4}=\frac{\tau\left[A \phi l_{t}^{\phi-1}\right]^{2}}{c_{t}^{2}\left\{\left[\frac{A^{2} \phi(\phi-1) l_{t}^{2 \phi-2}(1-\tau)+\tau\left[A \phi l_{t}^{\phi-1}\right]^{2}}{c_{t}}\right]-\left[\frac{A l_{t}^{\phi}(1-\tau)-m_{t} \theta}{c_{t}^{2}}\right]-\left[\frac{A l_{t}^{\phi} \beta}{l_{t}^{2}}\right]\right\}}
\end{aligned}
$$


Dadas las características de las funciones de utilidad y de producción con respecto al consumo y al trabajo (concavidad), se tiene que $v_{3}$ y $v_{4}$ son menores que cero. Lo anterior junto con la ecuación (37) conduce a otro importante resultado:

$$
\theta>-\left(r^{*}-\pi^{*}\right)
$$

lo que implica que la tasa óptima de crecimiento monetario tiene una caída respecto a la regla de completa liquidez de Friedman, es decir, $\theta=-\left(r^{*}-\pi^{*}\right)$. La razón de esto se puede ver en la restricción del gobierno en la forma

$$
\theta m_{t}+T_{t}+\tau A l_{t}^{\phi}=g_{t}+\left(r^{*}-\pi^{*}\right) a_{t}
$$

Al sustituir los niveles de equilibrio del ocio, de los saldos reales, del consumo, de los diferentes tipos de impuestos y del crédito neto, cuando se despeja de la ecuación (43) al gasto se obtiene su correspondiente trayectoria:

Grafica 3

Trayectoria del gasto de gobierno

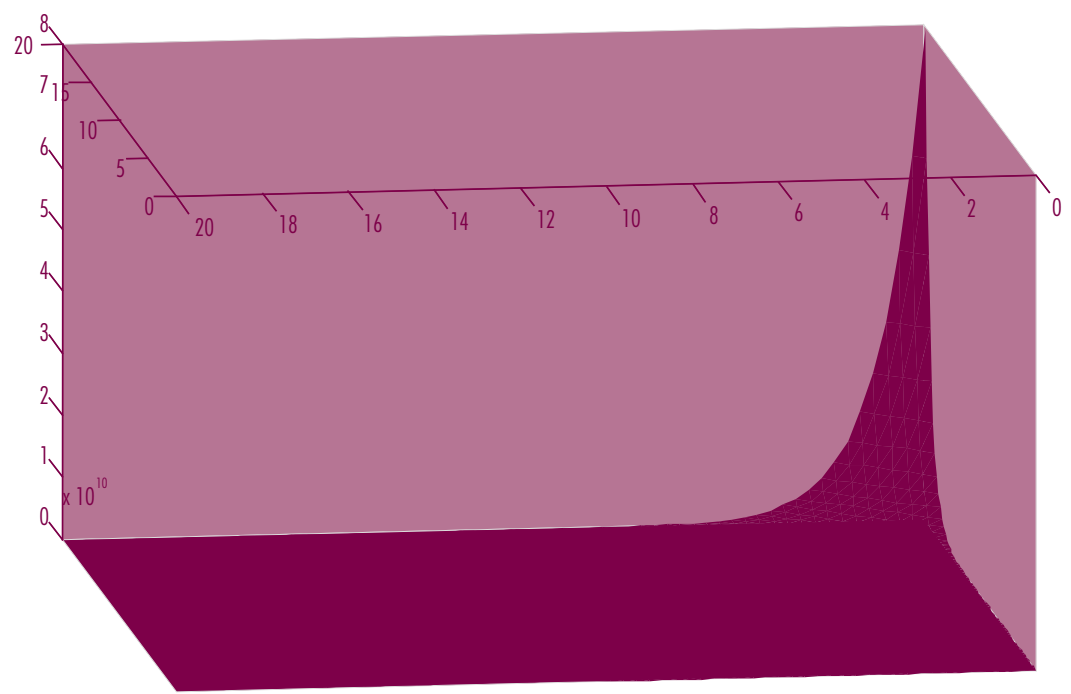

Fuente: Elaboración propia.

A diferencia de las variables de política fiscal del gobierno, es decir, el impuesto al ingreso y el impuesto de suma fija que puede generar distorsiones en la economía, el gasto de gobierno bajo la forma establecida en este modelo no genera distorsiones. 


\section{Conclusiones}

El diseño y aplicación de la política fiscal y monetaria es de suma importancia, sobre todo para garantizar que éstas no generen distorsiones sobre las decisiones de los individuos. Esta investigación ha desarrollado un modelo de agente representativo en una economía pequeña y abierta, con perfecta movilidad de capital, que ha permitido examinar las acciones del gobierno en materia fiscal y monetaria que son compatibles con las decisiones del sector privado.

Una conclusión relevante a la que se llega con el modelo propuesto es que un impuesto al ingreso afecta la liquidez y, en consecuencia, los individuos tienen que reducir su tenencia de saldos reales, los cuales deberían ser constantes para mantenerse en estado estacionario. Además la utilidad marginal que genera el gasto fiscal debe igualarse a la utilidad marginal generada por el consumo. Es importante destacar que no importa si las decisiones sobre las trayectorias de consumo y de trabajo son constantes en el tiempo o no, las políticas fiscal y monetaria necesariamente tienen que ser neutrales ante la liquidez para evitar la creación de distorsiones.

El modelo estudiado supone previsión perfecta, es decir, los individuos conocen el nivel general de precios en cada instante. Por supuesto, es necesario analizar el caso estocástico en donde los individuos conocen la distribución de probabilidad del nivel general de precios, situación que es más realista y que ya se considera en la agenda futura de investigación.

\section{Bibliografía}

Barro, R. J., "Government Spending in a Simple Model of Endogenous Growth", The Journal of Political Economy, vol. 98, núm. 5, 1990, pp. S103-S125.

Blanchard, O. y S. Fisher, Lectures on Macroeconomics, England, MIT Press, 1998.

Calvo, G. A., "On Models of Money and Perfect Foresight", International Economic Review, vol. 20, núm. 1, 1979, pp. 83-103.

Friedman, M., "Government Revenue from Inflation", Journal of Political Economy, vol. 79, núm. 4, jul.-Aug., 1971, pp. 846-856.

Inada, K. I., "On Neoclassical Models of Economic Growth", The Review of Economic Studies, vol. 32, núm. 2, 1965, pp. 151160.

Lockwood, B., M. Miller y L. Zhang, "Designing Monetary Policy when Unemploy- ment Persists", Economica, vol. 65, núm. 259, agosto de 1998, pp. 327-345.

Mankiw, G. y J. A. Miron, "The Changing Behavior of the Term Structure of Interest Rates", The Quarterly Journal of Economics, vol. 101, núm. 2, 1986, pp. 211-228.

Phelps, E. S., "Inflation in the theory of public finance", Swedish Journal of Economics, vol. 75, 1973, pp. 67-82.

Turnovsky, S. J., "Optimal Growth Accommodating Fiscal Policy in a Small Open Economy", Journal of International Money and Finance, vol. 6, 1987, pp. 179-193. , "Fiscal Policy and Growth in a Small Open Economy with Elastic Labor Supply", Canadian Journal of Economics, vol. 32, núm. 5, 1999.

, "Fiscal Policy, Elastic Labor Su- 


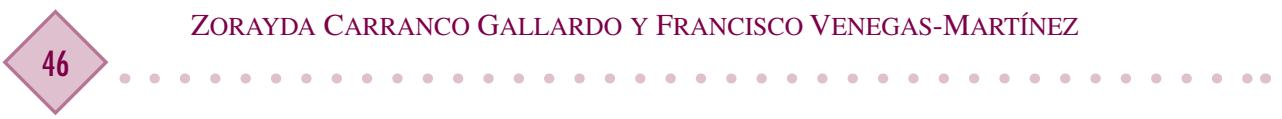

pply, and Endogenous Growth", Journal of Monetary Economics, vol. 5, 2000, pp. $185-210$

,"The Transitional Dynamics of Fiscal Policy: Long-Run Capital Accumulation and Growth", Journal of Money, Credit, and Banking, vol. 36, núm. 5, 2002.

y E. L. Grinols, "Risk, Optimal Government Finance and Monetary Policies in a Growing Economy", Economica, vol. 65, núm. 259, 1998, pp. 401-427.

Venegas-Martínez, F., "Utilidad, Aprendizaje y Estabilización", Gaceta de Economía, ITAM, año 5, núm. 10, 2000, pp. 153-169.
,"Temporary Stabilization: A Stochastic Analysis", Journal of Economic Dynamics and Control, vol. 25, núm. 9, 2001, pp. 1429-1449.

"Stochastic Temporary Stabilization: Undiversifiable Devaluation and Income Risks", Economic Modelling, vol. 23, núm. 1, 2006, pp. 157-173.

" "Temporary Stabilization in Developing Countries and the Real Option of Waiting when Consumption Can Be Delayed", International Journal of Economic Research, vol. 5, núm. 2, 2008 (por aparecer). 